La Plata-Th 95-10

\title{
SUPERGRAVITY AND A BOGOMOL'NYI BOUND IN THREE DIMENSIONS
}

\author{
José D. Edelstein*, Carlos Núñez and Fidel A. Schaposnik ${ }^{\dagger}$ \\ Departamento de Física, Universidad Nacional de La Plata \\ C.C. 67, (1900) La Plata \\ Argentina
}

\begin{abstract}
We discuss the $2+1$ dimensional Abelian Higgs model coupled to $N=2$ supergravity. We construct the supercharge algebra and, from it, we show that the mass of classical static solutions is bounded from below by the topological charge. As it happens in the global case, half of the supersymmetry is broken when the bound is attained and Bogomol'nyi equations, resulting from the unbroken supersymmetry, hold. These equations, which correspond to gravitating vortices, include a first order self-duality equation whose integrability condition reproduces the Einstein equation.
\end{abstract}

${ }^{*}$ CONICET

${ }^{\dagger}$ Investigador CICBA, Argentina 


\section{Introduction}

The relevance of the solutions to classical equations of motion of non-linear field theories (solitons, instantons) is nowadays recognized both in Mathematics and in Physics [1].

Since the pioneering work by Belavin, Polyakov, Schwartz and Tyupkin [2] an important feature of many of these (second order) equations of motion was discovered: through the obtention of a bound of topological origin (for the energy or the action, depending on the case), solutions can be found studying much simpler first order differential equations (self-duality equations or Bogomol'nyi equations [3]) An increasing number of works have been addressed to this issue in the last 20 years.

Already in ref.|⿰氵⿹丁口, where the Bogomol'nyi equations were first written for the Abelian Higgs model, it was recognized that the existence of these first-order equations was connected with the necessary conditions for supersymmetry in models with gauge symmetry breaking [5].

Other works then stressed this fact [6] but the main advance in the understanding of this question was achieved by Olive and Witten [7] in their work on the connection between topological quantum numbers and the central charge of extended supersymmetry. An important result of this investigation was to show that the classical aproximation to the mass spectrum is exact at the quantum level since supersymmetry ensures that there are no quantum corrections. In other words, the Bogomol'nyi's bound is valid quantum mechanically and is saturated.

Many models were studied afterwards following this line [8]-[11]. Concerning gauge theories with symmetry breaking (the case of interest in the present work) the interplay between Bogomol'nyi equations and supersymmetry can be understood as follows [11]:

For gauge theories with symmetry breaking having a topological charge and an $N=1$ supersymmetric version, the $N=2$ supersymmetric extension, which requires certain conditions on coupling constants, has a central charge coinciding with the topological charge. This relation ensures the existence, of a Bogomol'nyi bound and, consequently, of Bogomol'nyi equations.

It is important to note that in the soliton sector, half of the supersymmetries of the theory are broken 12 .

Once the connection between global supersymmetry and Bogomol'nyi 
bounds was understood, the natural question to pose is whether similar phenomena take place for local supersymmetry including gravity. That is, to investigate the possibility of establishing a connection between supergravity and Bogomol'nyi bounds for gravitating solitons and from this, to obtain firstorder differential equations whose solutions also solve Einstein and Maxwell (or Yang-Mills) equations together.

The works on this issue are based on those of Teitelboim [13], Deser and Teitelboim [14], Grisaru [15] and Witten-Nester-Israel [16]-[17] on positivity of the energy using supergravity. On this line, the Einstein-Maxwell theory was investigated by Gibbons and Hull [18 and the analog of Bogomol'nyi bounds were found for the $(\mathrm{ADM})$ mass $[19]$. In the same vein, Kallosh et al [20], Gibbons et al [21] and Cvetič et al [22] studied different gravity models.

Extending our previous work on the globally supersymmetric case [11], we study in the present paper the Abelian Higgs model coupled to supergravity in $2+1$ dimensions. We have chosen such a model in view of the experience accumulated in the study of Bogomol'nyi equations for vortex configurations, the connection we have already established for global supersymmetry and the simplicity one should expect from a $2+1$ abelian model. To our knowledge, there is not much work on this model except for a recent paper by Becker, Becker and Strominger [23], reported while the present investigation was in progress. Although some of our results overlap those in [23], we think it is worthwhile to present a detailed discussion of our approach which is based, as in the global case [11], in the study of the supersymmetry algebra and provides a systematic way of exploiting supersymmetry in the search of Bogomol'nyi equations. Not surprisingly, being the supersymmetry local, we find that a 1 form, analogous to the Witten-Nester-Israel form used in the proof of positivity of the energy in gravitation [16]-17, plays a central role in our derivation.

Indeed, we will show explicitely that the supercharge algebra can be realized in terms of the circulation, over a space-like surface contour, of a generalized Witten-Nester-Israel form adapted to the present $d=2+1$ case. This fact will be shown to be at the root of the existence of a Bogomol'nyi bound. Our procedure will be systematic in the sense that its formulation is adapted to any supergravity model where conserved supercharges could be defined and with a bosonic sector admiting topological charges.

The plan of the paper is as follows: in Section 2 we carefully discuss the $N=1, d=3+1$ supergravity action establishing our notation and conven- 
tions so that the dimensionally reduced $2+1$ Abelian Higgs model coupled to supergravity can be easily obtained (Section 3). Section 4 addresses to the supersymmetry algebra and its connection with the Bogomol'nyi bound (from which Bogomol'nyi equations can be obtained). We give a discussion of our results in Section 5.

\section{The $N=1$ Action in $d=4$}

We shall construct the $N=2$ locally supersymmetric Abelian Higgs model in 3-dimensional space by dimensional reduction [24] of an appropriate $N=1$, $d=4$ supergravity model. To our knowledge, it was not until very recently that the (Bosonic sector of the) corresponding Lagrangian has been written [23]. This section is addressed to the description of the Abelian Higgs model coupled to $N=1$ four-dimensional supergravity leaving for Section 3 the dimensional reduction.

Let us consider the following locally supersymmetric and gauge invariant superspace action for matter interacting with gravity and electromagnetism

$$
S=\int d^{4} x d^{4} \theta E\left[\frac{1}{2} \Delta\left(\Phi, \bar{\Phi} e^{2 \tilde{q} \mathcal{V}}\right) \exp \left(-\xi \kappa^{2} \mathcal{V}\right)+R e\left(\frac{2}{R} \bar{W} W\right)\right]
$$

Here, $\Phi$ is a chiral (matter) multiplet whose component fields are the Higgs field $\phi$, a Higgsino $\Xi$ and an auxilliary field $F . \mathcal{V}$ is a vector (gauge) multiplet which in the Wess-Zumino gauge contains the photon $A_{M}$, the photino $\Lambda$ and a real auxilliary field $D . W$ is the supercovariant strength multiplet containing the vector field strength. The superspace determinant is denoted by $E$ and $R$ is a chiral scalar curvature superfield [25]. We will distinguish curved $(M, N, R, \ldots)$ and flat $(A, B, C, \ldots)$ indices. The $N=1$ supergravity multiplet contains the vierbein $V_{M}^{A}$, the gravitino $\Psi_{M}$, a complex scalar auxilliary field $U$ and a pseudovector auxilliary field $b_{M} \cdot \Delta\left(\Phi, \bar{\Phi} e^{2 \tilde{q} \mathcal{V}}\right)$ is an arbitrary gauge invariant functional while $\tilde{q}$ is the $U(1)$ charge. $\xi$ is a real parameter and $\kappa$ is the gravitational constant. We have not included a superpotential term. The interaction between chiral and vector multiplets is the local version of the Fayet-Iliopoulos term [26]. We shall adopt hereafter the Wess-Zumino gauge.

After some calculations, the $N=1$ supergravity Lagrangian (11) can be 
written in terms of component fields as

$$
\mathcal{L}=\mathcal{L}_{\text {Bos }}+\mathcal{L}_{\text {Fer }}
$$

where

$$
\begin{aligned}
V^{-1} \mathcal{L}_{\text {Bos }} & =\frac{1}{6} \Upsilon \mathcal{R}-\frac{1}{4} g^{M R} g^{N S} F_{M N} F_{R S}-\frac{\partial^{2} \Upsilon}{\partial \phi^{*} \partial \phi}\left(D_{M} \phi\right)\left(D^{M} \phi\right)^{*} \\
& +\frac{\kappa}{3} b^{M}\left(i \frac{\partial \Upsilon}{\partial \phi} D_{M} \phi-i \frac{\partial \Upsilon}{\partial \phi^{*}}\left(D_{M} \phi\right)^{*}-\xi \kappa^{2} \Upsilon A_{M}\right) \\
& +\frac{\xi \kappa^{2}}{2} A^{M}\left(i \frac{\partial \Upsilon}{\partial \phi} D_{M} \phi-i \frac{\partial \Upsilon}{\partial \phi^{*}}\left(D_{M} \phi\right)^{*}-\frac{\xi \kappa^{2}}{2} \Upsilon A_{M}\right) \\
& +\frac{\kappa}{3}\left(U^{*} \frac{\partial \Upsilon}{\partial \phi} F+U \frac{\partial \Upsilon}{\partial \phi^{*}} F^{*}\right)+\frac{\partial^{2} \Upsilon}{\partial \phi^{*} \partial \phi}|F|^{2} \\
& +\frac{\kappa^{2}}{9} \Upsilon\left(|U|^{2}-b_{M} b^{M}\right)+\tilde{q} D \phi \frac{\partial \Upsilon}{\partial \phi}-\frac{\xi \kappa^{2}}{2} \Upsilon D+\frac{1}{2} D^{2}
\end{aligned}
$$

and

$$
\begin{aligned}
V^{-1} \mathcal{L}_{F e r} & =\frac{\kappa^{2}}{12} \Upsilon \frac{\epsilon^{M N R S}}{V} \bar{\Psi}_{M} \Gamma_{5} \Gamma_{N}\left(\mathcal{D}_{R}+i \frac{3 \xi \kappa^{2}}{4} A_{R} \Gamma_{5}\right) \Psi_{S} \\
& -\frac{\partial^{2} \Upsilon}{\partial \phi^{*} \partial \phi} \bar{\Xi}_{-}\left(\not D+i \frac{\kappa}{6} \not b-i \frac{\xi \kappa^{2}}{2} \not A\right) \Xi_{+}-\frac{1}{4} \bar{\Lambda}\left(\hat{D}+i \frac{\kappa}{2} \not \Gamma_{5}\right) \Lambda \\
& +\frac{\partial^{3} \Upsilon}{\partial \phi^{*} \partial \phi^{2}} \bar{\Xi}_{-} \not D \phi \Xi_{+}+\frac{1}{2} \frac{\partial^{4} \Upsilon}{\partial \phi^{*^{2}} \partial \phi^{2}} \bar{\Xi}_{-} \Xi_{-} \bar{\Xi}_{+} \Xi_{+}-\frac{\partial^{3} \Upsilon}{\partial \phi^{*} \partial \phi^{2}} \bar{\Xi}_{-} \Xi_{-} F^{*} \\
& -\frac{i \kappa^{2}}{3} b^{M} \frac{\partial \Upsilon}{\partial \phi} \bar{\Psi}_{M-} \Xi_{-}+\frac{i}{8} \xi \kappa^{3} \Upsilon \bar{\Psi} \cdot \Gamma \Gamma_{5} \Lambda-\frac{\kappa}{3} U^{*} \frac{\partial^{2} \Upsilon}{\partial \phi^{2}} \Xi_{-} \Xi_{-} \\
& +\kappa \frac{\partial^{2} \Upsilon}{\partial \phi^{*} \partial \phi} \bar{\Psi}_{M-}(\not D \phi)^{*} \Gamma_{M} \Xi_{-}-\frac{4 \kappa}{3} \frac{\partial \Upsilon}{\partial \phi} \bar{\Xi}_{-} \Sigma^{M N} \mathcal{D}_{M} \Psi_{N-} \\
& -\frac{\kappa^{2}}{8} \frac{\epsilon^{M N R S}}{V} \bar{\Psi}_{M} \Gamma_{N} \Psi_{R}\left(\frac{\partial \Upsilon}{\partial \phi} D_{S} \phi+\frac{1}{2} \frac{\partial^{2} \Upsilon}{\partial \phi^{*} \partial \phi} \bar{\Xi}_{+} \Gamma_{S} \Xi_{-}\right) \\
& +\frac{\kappa^{3}}{6} \frac{\partial \Upsilon}{\partial \phi} \bar{\Xi}_{-}\left(\Psi_{M-} \bar{\Psi}_{1} \Gamma \Psi^{M}+\Sigma^{M N}\left(\frac{1}{2} \Psi_{-}^{R} \bar{\Psi}_{N} \Gamma_{R} \Psi_{M}\right.\right. \\
& \left.\left.+\Psi_{N-} \bar{\Psi}_{M} \Gamma \cdot \Psi\right)\right)+\frac{i}{2} \tilde{q} \frac{\partial \Upsilon}{\partial \phi} \phi \bar{\Psi}_{-} \cdot \Gamma \Lambda_{+}-2 i \tilde{q} \frac{\partial^{2} \Upsilon}{\partial \phi^{*} \partial \phi} \phi \bar{\Lambda}_{+} \Xi_{+}
\end{aligned}
$$




$$
\begin{aligned}
& -\frac{\kappa^{2}}{2} \frac{\partial^{2} \Upsilon}{\partial \phi^{*} \partial \phi} \bar{\Psi}_{M-} \Xi_{-} \bar{\Psi}^{M}{ }_{+} \Xi_{+}-\frac{\xi \kappa^{2}}{2} \frac{\partial \Upsilon}{\partial \phi^{*}} \Xi_{+} \Lambda_{+} \\
& +\frac{\kappa}{8} \bar{\Psi}_{T} \Sigma^{M N} \Gamma^{T} \Lambda F_{M N}+\text { h.c. }
\end{aligned}
$$

Here $V$ is the determinant of the vierbein,

$$
\left.V(x) \equiv E(x, \theta, \bar{\theta})\right|_{\theta=\bar{\theta}=0},
$$

and $\mathcal{R}$ is the Ricci scalar (depending on the vierbein $V_{M}^{A}$ and the spinorial connection $\left.\Omega_{M A B}\right)$. $\Upsilon$ is a functional of the Higgs field defined as:

$$
\left.\Upsilon\left(\phi, \phi^{*}\right) \equiv \Delta\left(\Phi, \bar{\Phi} e^{2 \tilde{q} \mathcal{V}}\right)\right|_{\theta=\bar{\theta}=0},
$$

subindices "-" and "+" indicate left or right-handed fermions and the "hatted" derivative acting on the photino $\Lambda$ is defined as

$$
\hat{D}_{M} \Lambda=\mathcal{D}_{M} \Lambda+\frac{\kappa}{4}\left(\partial_{R} A_{S}-\frac{\kappa}{2} \bar{\Psi}_{R} \Gamma_{S} \Lambda-(R \leftrightarrow S)\right) \Sigma^{R S} \Psi_{M}
$$

where $\mathcal{D}_{M}$ is the covariant derivative over fermionic fields:

$$
\mathcal{D}_{M}=\partial_{M}+\frac{1}{4} \Omega_{M A B} \Sigma^{A B}
$$

Finally,

$$
\not D \Xi_{ \pm}=\not D \Xi_{ \pm}-i \tilde{q} \not A \Xi_{ \pm}
$$

is the Maxwell and general coordinate covariant derivative acting on $\Xi$. Let us mention that a Weyl scaling of vierbein and fermion fields and a particular choice of the (up to now arbitrary) functional $\Upsilon$ is necessary in order to bring (2) to its canonical form.

Lagrangian (2) is invariant under the following set of local supersymmetry transformations (with anticommuting parameter $\epsilon(x))$ :

$$
\begin{aligned}
\delta A_{M} & =-\frac{1}{2} \bar{\epsilon}_{-} \Gamma_{M} \Lambda_{+}-\frac{1}{2} \bar{\epsilon}_{+} \Gamma_{M} \Lambda_{-} \quad, \quad \delta V_{M}^{A}=\frac{\kappa}{2} \bar{\epsilon}_{-} \Gamma^{A} \Psi_{M+}+\frac{\kappa}{2} \bar{\epsilon}_{+} \Gamma^{A} \Psi_{M-} \\
\delta D & =\frac{1}{2} \bar{\epsilon}_{-}\left(\hat{\not D}-\frac{i \kappa}{2} \not b\right) \Lambda_{+}-\frac{i}{2} \bar{\epsilon}_{+}\left(\hat{\not D}+\frac{i \kappa}{2} \not b\right) \Lambda_{-} \quad, \quad \delta U=\frac{1}{2} \bar{\epsilon}_{+} \Gamma \cdot \hat{R}_{-} \\
\delta F & =\bar{\epsilon}_{+}\left(\hat{\not D}-\frac{i \kappa}{2} \not b\right) \Xi_{-}-\frac{\kappa}{3} \bar{\Xi}_{-}\left(U \epsilon_{-}+i \not b \epsilon_{+}\right)+i \tilde{q} \bar{\epsilon}_{+} \Lambda \phi \quad, \quad \delta \phi=\bar{\epsilon}_{-} \Xi_{-}
\end{aligned}
$$




$$
\begin{gathered}
\delta b_{M}=\frac{3 i}{4} \bar{\epsilon}_{-}\left(\hat{R}_{M-}-\frac{1}{3} \Gamma_{M} \Gamma \cdot \hat{R}_{-}\right)+h . c . \\
\delta \Lambda_{+}=\frac{1}{2}\left(\partial_{M} A_{N}-\frac{\kappa}{2} \bar{\Psi}_{M} \Gamma_{N} \Lambda-(M \leftrightarrow N)\right) \Sigma^{M N} \epsilon_{+}-\frac{i}{2} D \epsilon_{+} \\
\delta \Xi_{-}=\frac{1}{2}\left(\not D \phi-\kappa \Gamma^{M} \bar{\Psi}_{M-} \Xi_{-}\right) \epsilon_{+}+\frac{1}{2} F \epsilon_{-} \\
\delta \Psi_{M-}=\frac{2}{\kappa}\left(\mathcal{D}_{M}+\frac{i \kappa}{2} b_{M}\right) \epsilon_{-}+\frac{1}{6} \Gamma_{M}\left(U^{*} \epsilon_{+}-i \not b \epsilon_{-}\right)
\end{gathered}
$$

where $\hat{R}_{M-}$ is given by:

$$
\hat{R}_{M-}=i \frac{\epsilon^{M N R T}}{V} \Gamma_{5} \Gamma_{N}\left[\mathcal{D}_{R}+\frac{i \kappa}{2} \Gamma_{5} b_{R}+\frac{\kappa}{6} \Gamma_{R}\left(U-i \not b \Gamma_{5}\right)\right] \Psi_{T}
$$

and

$$
\hat{D}_{M} \Xi_{ \pm}=\mathcal{D}_{M} \Xi_{ \pm}-i \tilde{q} A_{M} \Xi_{ \pm} \pm \frac{i \kappa}{2} b_{M} \Xi_{ \pm}-\frac{\kappa}{2}\left((\hat{\not D} \phi) \Psi_{M-}+F \Psi_{M+}\right)
$$

We eliminate auxiliary fields and compute on-shell quantities, the fermion Lagrangian (4) becomes:

$$
\begin{aligned}
V^{-1} \mathcal{L}_{F e r} & =\frac{\kappa^{2}}{6} \Upsilon \frac{\epsilon^{M N R S}}{V} \bar{\Psi}_{M} \Gamma_{5} \Gamma_{N}\left(\mathcal{D}_{R}+i \frac{3 \xi \kappa^{2}}{4} A_{R} \Gamma_{5}\right) \Psi_{S} \\
& -\frac{1}{2} \bar{\Lambda}\left(\hat{\not D}-i \frac{3 \xi \kappa^{2}}{4} \not A \Gamma_{5}\right) \Lambda-\frac{4 \kappa}{3}\left(\frac{\partial \Upsilon}{\partial \phi} \bar{\Xi}_{-} \Sigma^{M N} \mathcal{D}_{M} \Psi_{N-}+\text { h.c. }\right) \\
& -\frac{\partial^{2} \Upsilon}{\partial \phi^{*} \partial \phi} \bar{\Xi}\left(\not D-i\left(\tilde{q}+\frac{3 \xi \kappa^{2}}{4}\right) \not A\right) \Xi+V^{-1} \mathcal{L}_{F e r}^{i n t}
\end{aligned}
$$

where $\mathcal{L}_{\text {Fer }}^{\text {int }}$ contains complicated interactions involving fermion fields whose explicit form is not of interest for us. It is interesting to stress the occurrence of a charge $3 \xi \kappa^{2} / 4$ for all fermion fields (originated in the Fayet-Iliopoulos term).

We shall often have to put all fermion fields to zero. Given a functional $Q$ depending both on bosonic and fermionic fields, it will then be convenient to define $Q \mid$ for

$$
Q|\equiv Q|_{\Psi_{A}, \Lambda, \Xi=0} .
$$


Under condition (10) the only surviving supersymmetric transformations are those corresponding to fermionic fields:

$$
\begin{gathered}
\delta \Lambda_{+}\left|=\frac{1}{2} F_{M N} \Sigma^{M N} \epsilon_{+}-\frac{i}{2}\left(\frac{\xi \kappa^{2}}{2} \Upsilon-\tilde{q} \phi \frac{\partial \Upsilon}{\partial \phi}\right) \epsilon_{+} \quad, \quad \delta \Xi_{-}\right|=\frac{1}{2}(\not D \phi) \epsilon_{+} \\
\delta \Psi_{M-} \mid=\frac{2}{\kappa}\left(\mathcal{D}_{M}+\frac{3 i}{4 \Upsilon}\left[i \frac{\partial \Upsilon}{\partial \phi}\left(D_{M} \phi\right)-i \frac{\partial \Upsilon}{\partial \phi^{*}}\left(D_{M} \phi\right)^{*}-\xi \kappa^{2} \Upsilon A_{M}\right]\right) \epsilon_{-} \\
-\frac{i}{4 \kappa \Upsilon} \Gamma_{M}\left[i \frac{\partial \Upsilon}{\partial \phi}(\not D \phi)-i \frac{\partial \Upsilon}{\partial \phi^{*}}(\not D \phi)^{*}-\xi \kappa^{2} \Upsilon \not A\right] \epsilon_{-}
\end{gathered}
$$

Concerning the Lagrangian, it becomes

$$
\begin{aligned}
V^{-1} \mathcal{L} \mid & =\frac{1}{6} \Upsilon \mathcal{R}-\frac{\partial^{2} \Upsilon}{\partial \phi^{*} \partial \phi}\left(D_{M} \phi\right)\left(D^{M} \phi\right)^{*}-\frac{1}{4} g^{M R} g^{N S} F_{M N} F_{R S} \\
& +\frac{1}{4 \Upsilon}\left(i \frac{\partial \Upsilon}{\partial \phi} D_{M} \phi-i \frac{\partial \Upsilon}{\partial \phi^{*}}\left(D_{M} \phi\right)^{*}-\xi \kappa^{2} \Upsilon A_{M}\right)^{2} \\
& -\frac{1}{2}\left(\tilde{q} \phi \frac{\partial \Upsilon}{\partial \phi}-\frac{1}{2} \xi \kappa^{2} \Upsilon\right)^{2}
\end{aligned}
$$

In order to bring (12) to its canonical way one can perform a Weyl rescaling on the Vierbein

$$
\begin{gathered}
V_{M}^{A} \rightarrow V_{M}^{A}\left(-\frac{3}{\kappa^{2} \Upsilon}\right)^{1 / 2} \equiv V_{M}^{A} e^{-\frac{1}{6} \mathcal{J}\left(\phi, \phi^{*}\right)} \\
g^{M N} \rightarrow g^{M N}\left(-\frac{\kappa^{2} \Upsilon}{3}\right) \quad, \quad V \rightarrow \frac{9}{\kappa^{4} \Upsilon^{2}} V
\end{gathered}
$$

where

$$
\mathcal{J}\left(\phi, \phi^{*}\right) \equiv 3 \log \left(-\frac{\kappa^{2} \Upsilon}{3}\right)
$$

If we now choose $2 \mathcal{J}\left(\phi, \phi^{*}\right)=-\kappa^{2} \phi \phi^{*}$ and redefine $\xi=-\tilde{q} v^{2} / 3$, the Lagrangian takes the form

$$
\begin{aligned}
V^{-1} L \mid & =-\frac{1}{2 \kappa^{2}} \mathcal{R}-\frac{1}{4} g^{M R} g^{N S} F_{M N} F_{R S}-\frac{1}{2}\left(D_{M} \phi\right)\left(D^{M} \phi\right)^{*} \\
& -\frac{\tilde{q}^{2}}{8}\left(|\phi|^{2}-v^{2}\right)^{2}
\end{aligned}
$$


which is the expected Abelian Higgs model Lagrangian minimally coupled to gravity. Note that the coupling constant of the Higgs potential is related to the electric charge by the well-known condition

$$
\lambda=\frac{\tilde{q}^{2}}{8}
$$

This condition was originally found for the globally supersymmetric model [5]. As explained in [11] it gives a necessary condition for extending $N=1$ to $N=2$ supersymmetry and, at the same time, for finding a Bogomol'nyi bound [3, 田 for the energy of the Abelian Higgs model.

It is interesting to note that Weyl transformations for fermion fields, in correspondance with (13) bring, on the one hand the gravitino Lagrangian to its usual Rarita-Schwinger form. On the other hand, as we shall see, the Higgs potential and the Higgs current take after the scaling its usual form as can be seen in the resulting Bogomol'nyi equations. Under this Weyl scaling

$$
\begin{aligned}
\Psi_{M} \rightarrow\left(-\frac{3}{\kappa^{2} \Upsilon}\right)^{1 / 4} \Psi_{M} \quad, \quad \Lambda & \rightarrow\left(-\frac{3}{\kappa^{2} \Upsilon}\right)^{-3 / 4} \Lambda \quad, \quad \Xi \rightarrow\left(-\frac{3}{\kappa^{2} \Upsilon}\right)^{-1 / 4} \Xi \\
\epsilon & \rightarrow\left(-\frac{3}{\kappa^{2} \Upsilon}\right)^{1 / 4} \epsilon
\end{aligned}
$$

one has

$$
\begin{gathered}
\delta \Lambda_{+}\left|=\frac{1}{2} F_{M N} \Sigma^{M N} \epsilon_{+}+\frac{i \tilde{q}}{4}\left(|\phi|^{2}-v^{2}\right) \epsilon_{+} \quad, \quad \delta \Xi_{-}\right|=\frac{1}{2}(\not D \phi) \epsilon_{+} \\
\delta \Psi_{M-} \mid=\frac{2}{\kappa}\left(\mathcal{D}_{M}+\frac{i \kappa^{2}}{4}\left(J_{M}+\tilde{q} v^{2} A_{M}\right)\right) \epsilon_{-}
\end{gathered}
$$

where

$$
J_{M}=\frac{i}{2}\left(\phi\left(D_{M} \phi\right)^{*}-\phi^{*}\left(D_{M} \phi\right)\right)
$$

is the Higgs field current.

Although we will finally put all fermion fields to zero, we will need for further calculations the explicit form of the kinetic Lagrangian for fermions. After the Weyl rescaling (18) is performed in Lagrangian (9), one can note that the kinetic fermionic part can be diagonalized by the following shift:

$$
\Psi_{M-} \rightarrow \Psi_{M-}-\frac{1}{\kappa \Upsilon} \frac{\partial \Upsilon}{\partial \phi^{*}} \Gamma_{M} \Xi_{+}
$$


such that the Fermion Lagrangian can be finally written as:

$$
\begin{aligned}
V^{-1} L_{F e r} & =-\frac{1}{2} \epsilon^{M N R S} V \bar{\Psi}_{M} \Gamma_{5} \Gamma_{N}\left(\mathcal{D}_{R}+i \frac{\tilde{q} v^{2} \kappa^{2}}{4} A_{R} \Gamma_{5}\right) \Psi_{S} \\
& -\frac{1}{2} \bar{\Xi}\left(\not D-i\left(\tilde{q}+\frac{\tilde{q} v^{2} \kappa^{2}}{4}\right) \not A\right) \Xi \\
& -\frac{1}{2} \bar{\Lambda}\left(\not D-i \frac{\tilde{q} v^{2} \kappa^{2}}{4} \not A \Gamma_{5}\right) \Lambda+V^{-1} \tilde{\mathcal{L}}_{F e r}^{i n t}
\end{aligned}
$$

once the above mentioned conditions for $\mathcal{J}\left(\phi, \phi^{*}\right)$ and $\xi$ are adopted.

\section{Dimensional Reduction and Extended su- persymmetry}

We shall now derive the $d=3, N=2$ Lagrangian by dimensional reduction of (16). To this end, we write the vierbein as:

$$
V_{M}^{A}=\left(\begin{array}{cc}
e_{\mu}^{a} & a_{\mu} \\
0 & \varphi
\end{array}\right)
$$

(we use $\mu=0,1,2$ for curved coordinates and $a=0,1,2$ for flat indices) and suppose that whole set of fields are $x_{3}$-independent. We will accordingly choose $x_{3}$ as the variable which is eliminated within the dimensional

reduction procedure. We introduce in (23) $e_{\mu}^{a}$ as the dreibein of the reduced 3-dimensional manifold, $a_{\mu}$ as a vector field and $\varphi$ as a real scalar. We have chosen the gauge $V_{3}^{a}=0$, which can always be attained by a suitable local Lorentz transformation [24]. Indeed, an infinitesimal field variation of $V_{M}^{A}$ under local supersymmetry, local Lorentz and general coordinate transformations reads

$$
\delta V_{M}^{A}=-i \kappa \bar{\epsilon} \Gamma^{A} \Psi_{M}+\omega_{B}^{A} V_{M}^{B}+\partial_{M} \eta^{R} V_{R}^{A}+\eta^{R} \partial_{R} V_{M}^{A}
$$

where $\bar{\epsilon}, \omega_{B}^{A}$ and $\eta^{R}$ are the corresponding local parameters. Freedom associated with general coordinate invariance in four dimensions can be exploited to put

$$
a_{\mu}=0 \quad, \quad \varphi=1
$$


without spoiling the local Einstein group of the reduced 3-dimensional spacetime.

With these conditions, the metric tensor reads

$$
g^{M N}=V_{A}^{M} V_{B}^{N} \eta^{A B}=\left(\begin{array}{cc}
g^{\mu \nu} & 0 \\
0 & -1
\end{array}\right)
$$

where $\eta^{A B}=\operatorname{diag}(+---)$. Concerning the spinorial connection, it takes the form

$$
\Omega_{c a b}\left|=\omega_{c a b}\right|=-\frac{1}{2}\left(e_{c}^{m} e_{a}^{n}-e_{a}^{m} e_{c}^{n}\right) \partial_{n} e_{m b}+\frac{1}{2} e_{a}^{m} e_{b}^{n} \partial_{n} e_{m c}-(a \leftrightarrow b)
$$

while all other components vanish. Then, the Ricci scalar in four dimensions $\mathcal{R}$ reduces to the corresponding one in $d=3$, which will be denote as $R$.

After dimensional reduction, Lagrangian (16) becomes

$$
\begin{aligned}
e^{-1} L \mid & =-\frac{1}{2 \kappa^{2}} R-\frac{1}{4} g^{\mu \rho} g^{\nu \sigma} F_{\mu \nu} F_{\rho \sigma}-\frac{1}{2}\left(D_{\mu} \phi\right)\left(D^{\mu} \phi\right)^{*} \\
& +\frac{1}{2} \partial_{\mu} S \partial^{\mu} S+\frac{\tilde{q}^{2}}{2} S^{2}|\phi|^{2}-\frac{\tilde{q}^{2}}{8}\left(|\phi|^{2}-v^{2}\right)^{2}
\end{aligned}
$$

where we have identified

$$
A_{M} \equiv\left(A_{\mu}, S\right) \text {. }
$$

Lagrangian (28) describes the dynamics of the Bosonic sector for the $d=3$ Abelian Higgs model coupled to $N=2$ supergravity. We will now focus on the dimensional reduction of supersymmetric transformation rules written in eq.(19). To this end, let us specify our representation for the Clifford algebra

$$
\begin{gathered}
\Gamma^{a}=\gamma^{a} \otimes \tau_{3} \quad, \quad \Gamma^{3}=1 \otimes i \tau_{2} \quad, \quad \Gamma^{5}=1 \otimes \tau_{1} \\
\Sigma^{a b}=\sigma^{a b} \otimes 1 \quad, \quad \Sigma^{a 3}=-\Sigma^{3 a}=\gamma^{a} \otimes \tau_{1}
\end{gathered}
$$

where $\gamma^{a}$ are $2 \times 2$ Dirac matrices for $d=3$ and $\sigma^{a b}=1 / 2\left[\gamma^{a}, \gamma^{b}\right]$. In this basis the original 4-dimensional Majorana spinors take the form

$$
\Psi=\left(\begin{array}{c}
\Psi_{1} \\
i \Psi_{2}
\end{array}\right)
$$


where $\Psi_{1}$ and $\Psi_{2}$ are 2-component real spinors which can be taken as 3dimensional Majorana fields. Finally, with these Majorana fields one can construct a Dirac spinor $\psi$ in 3 dimensions

$$
\psi=\Psi_{1}+i \Psi_{2}
$$

The dimensionally reduced supersymmetric transformations for fermions read

$$
\begin{gathered}
\delta \lambda \mid=\frac{1}{2} F_{\mu \nu} \sigma^{\mu \nu} \epsilon+\frac{i \tilde{q}}{4}\left(|\phi|^{2}-v^{2}\right) \epsilon+\gamma^{\mu} \partial_{\mu} S \epsilon \\
\delta \chi\left|=\frac{1}{2}(\not D \phi+i \tilde{q} S \phi) \epsilon \quad, \quad \delta \psi_{3}\right|=-i \frac{\tilde{q} \kappa}{2} S\left(|\phi|^{2}-v^{2}\right) \epsilon \\
\delta \psi_{\mu} \mid=\frac{2}{\kappa}\left(\mathcal{D}_{\mu}+i \frac{\kappa^{2}}{4}\left(J_{\mu}+\tilde{q} v^{2} A_{\mu}\right)\right) \epsilon \equiv \frac{2}{\kappa} \hat{\nabla}_{\mu} \epsilon .
\end{gathered}
$$

Note that we have included the transformation for $\psi_{3}$, a remnant of the 4 dimensional starting model. However, as we shall see, $S$ will be put to zero to recover the Abelian Higgs model in the bosonic sector, this giving a trivial $\psi_{3}$ transformation rule. Being $\epsilon$ a Dirac fermion, transformations (31) are $N=2$ supersymmetric ones. Supersymmetric covariant derivative $\nabla_{\mu}$ is defined as

$$
\hat{\nabla}_{\mu} \epsilon=\left(\partial_{\mu}+\frac{1}{4} \omega_{\mu a b} \sigma^{a b}+i \frac{\kappa^{2}}{4}\left(J_{\mu}+\tilde{q} v^{2} A_{\mu}\right)\right) \epsilon
$$

We shall end this Section by performing the dimensional reduction of the fermionic Lagrangian (22) which is the fermionic counterpart of the $N=2$ bosonic Lagrangian (28). In order to achieve a diagonalized kinetic fermionic sector, we perform the following shift

$$
\psi_{\mu} \rightarrow \psi_{\mu}+i \gamma_{\mu} \psi_{3}
$$

after which, the resulting fermionic Lagrangian reads:

$$
\begin{aligned}
e^{-1} L_{F e r} & =-\frac{1}{2} \frac{\epsilon^{\rho \mu \sigma}}{e} \bar{\psi}_{\rho} \partial_{\mu} \psi_{\sigma}-\frac{1}{2} \bar{\lambda} \not \partial \lambda-\frac{1}{2} \bar{\chi} \not \partial \chi \\
& -i \bar{\psi}_{3} \not \partial \psi_{3}+e^{-1} \hat{L}_{F e r}^{i n t}
\end{aligned}
$$

where the last term $\hat{L}_{F e r}^{i n t}$ includes also gauge interactions. 


\section{Self-duality equations and the Bogomol'nyi bound}

In this Section we shall obtain a Bogomol'nyi bound for field configurations in the $d=3$ Abelian Higgs model coupled to gravity. In doing this, we shall

make explicit the relation between this bound and the supercharge algebra of the $N=2$ theory.

The equations of motion for bosonic matter fields are:

$$
\begin{gathered}
\frac{1}{\sqrt{-g}} \partial_{\mu}\left(\sqrt{-g} F^{\mu \nu}\right)=-\tilde{q} J^{\nu} \\
\frac{1}{\sqrt{-g}} D_{\mu}\left(\sqrt{-g} D^{\mu} \phi\right)=\frac{\tilde{q}^{2}}{2}\left(|\phi|^{2}-v^{2}\right) \phi-\tilde{q}^{2} S^{2} \phi \\
\frac{1}{\sqrt{-g}} \partial_{\mu}\left(\sqrt{-g} \partial^{\mu} S\right)=\tilde{q}^{2}|\phi|^{2} S .
\end{gathered}
$$

Concerning Einstein equations

$$
\begin{gathered}
R_{\mu \nu}-\frac{1}{2} g_{\mu \nu} R=T_{\mu \nu}^{m a t} \\
T_{\mu \nu}^{m a t}=-g^{\lambda \tau} F_{\mu \tau} F_{\nu \lambda}-\frac{1}{2}\left(D_{\mu} \phi\right)\left(D_{\nu} \phi\right)^{*}-\frac{1}{2}\left(D_{\mu} \phi\right)^{*}\left(D_{\nu} \phi\right)-\partial_{\mu} S \partial_{\nu} S \\
+g_{\mu \nu}\left[\frac{1}{4} F_{\rho \sigma} F^{\rho \sigma}+\frac{1}{2}\left(D_{\rho} \phi\right)\left(D^{\rho} \phi\right)^{*}-\frac{1}{2} \partial_{\rho} S \partial^{\rho} S-\frac{\tilde{q}^{2}}{2} S^{2}|\phi|^{2}\right. \\
\left.+\frac{\tilde{q}^{2}}{8}\left(|\phi|^{2}-v^{2}\right)^{2}\right]
\end{gathered}
$$

Since we shall focus on the Abelian Higgs model coupled to gravity, we make at this point $S=0$. Moreover, since Bogomol'nyi equations correspond to static configurations with $A_{0}=0$, we also impose these conditions (note that in this case $T_{0 i}^{\text {mat }}=0$ ).

Concerning the metric, let us notice that a static spacetime admits a surface $\Pi$ orthogonal everywhere to the time-like killing vector field $\frac{\partial}{\partial t}$. In a local chart adapted to $\frac{\partial}{\partial t}$, the line element can be written as:

$$
d s^{2}=d t^{2}+g_{i j} d x^{i} d x^{j}
$$


where $g_{i j}$ is a function depending only on spatial variables that span $\Pi$. Now, it is well-known that any 2-dimensional metric is Kähler and then we write the interval in the form

$$
d s^{2}=d t^{2}-\Omega^{2} d z d \bar{z}
$$

where $\Omega$ is a function of the conformal coordinates $\Omega(z, \bar{z})$. Note that for any finite energy configuration, Einstein equations (37) constrain the asymptotic behaviour of $\Omega$ to be

$$
\Omega(z, \bar{z}) \rightarrow(z \bar{z})^{-\frac{\kappa^{2} M}{2}}
$$

so that the metric is asymptotic to a flat cone with deficit angle $\delta=\kappa^{2} M$ [27], $M$ being related to the source mass

$$
\begin{aligned}
M & =\frac{1}{4 \pi} \int d z d \bar{z} \Omega^{2} T_{t t}^{m a t}=\frac{1}{4 \pi} \int d z d \bar{z} \Omega^{2}\left[\frac{1}{2} F_{z \bar{z}} F^{z \bar{z}}\right. \\
& \left.+\frac{1}{2}\left(D_{z} \phi\right)\left(D^{z} \phi\right)^{*}+\frac{1}{2}\left(D_{\bar{z}} \phi\right)\left(D^{\bar{z}} \phi\right)^{*}+\frac{\tilde{q}^{2}}{8}\left(|\phi|^{2}-v^{2}\right)^{2}\right] .
\end{aligned}
$$

The spacetime metric (40) can be generated by the following dreibein

$$
e_{t}^{0}=e_{\bar{z}}^{-}=1 \quad, \quad e_{z}^{+}=\Omega^{2}
$$

(all the other components vanishing) with the flat metric written in conformal coordinates as

$$
\eta_{00}=-2 \eta_{+-}=-2 \eta_{-+}=1 .
$$

The non-vanishing component of the spinorial connection is:

$$
\omega_{\bar{z}+-}=-\bar{\partial} \log \Omega
$$

(here $\partial \equiv \partial_{z}$ and $\bar{\partial} \equiv \partial_{\bar{z}}$ ).

We shall now analyse the $N=2$ algebra of supercharges for our model. To construct these charges we shall follow the Noether method. The conserved current associated with local supersymmetry is given by:

$$
\mathcal{J}^{\mu}[\epsilon]=\sum_{\Phi} \frac{\delta L}{\delta \nabla_{\mu} \Phi} \delta_{\epsilon} \Phi+\sum_{\Psi} \frac{\delta L}{\delta \nabla_{\mu} \Psi} \delta_{\epsilon} \Psi-\theta^{\mu}[\epsilon]
$$

where $\Phi$ and $\Psi$ represent the whole set of bosonic and fermionic fields respectively. Concerning $\theta^{\mu}[\epsilon]$, it is defined through

$$
\delta_{\epsilon} S=\int d^{3} x \nabla_{\mu} \theta_{\epsilon}^{\mu}
$$


In the present case we have:

$$
\begin{aligned}
\theta^{\mu}[\epsilon] & =-\frac{3}{2 \kappa} \frac{\epsilon^{\mu \rho \sigma}}{e} \bar{\psi}_{\rho} \hat{\nabla}_{\sigma} \epsilon-\frac{1}{2} \bar{\epsilon}\left(D_{\mu} \phi\right) \chi+\frac{1}{2} \bar{\epsilon} \gamma_{\nu} F^{\mu \nu} \lambda-\frac{1}{8} \bar{\chi} \gamma^{\mu}(\not D \phi) \\
& -\frac{1}{4} \bar{\lambda} \gamma^{\mu}\left(\frac{1}{2} F_{\mu \nu} \sigma^{\mu \nu}+\frac{i \tilde{q}}{4}\left(|\phi|^{2}-v^{2}\right)\right) \epsilon+\text { h.c. }+\ldots
\end{aligned}
$$

where the dots indicate terms containing products of three fermion fields, which are not relevant for our construction since, after computing the supercharge algebra, we will put all fermions to zero. Inserting $\theta^{\mu}$ in (45) we obtain the following expression for the supersymmetry current:

$$
\begin{aligned}
\mathcal{J}^{\mu}[\epsilon] & =-\bar{\lambda} \gamma^{\mu}\left(\frac{1}{2} F_{\alpha \beta} \sigma^{\alpha \beta}+\frac{i \tilde{q}}{4}\left(|\phi|^{2}-v^{2}\right)\right) \epsilon-\frac{1}{2} \bar{\chi} \gamma^{\mu}(\not D \phi) \epsilon \\
& -\frac{2}{\kappa} \frac{\epsilon^{\rho \mu \sigma}}{e} \bar{\psi}_{\rho} \hat{\nabla}_{\sigma} \epsilon+\text { h.c. }+\ldots
\end{aligned}
$$

The conserved charges associated with (48),

$$
\mathcal{Q}[\epsilon]=\int_{\Sigma} \mathcal{J}^{\mu}[\epsilon] d \Sigma_{\mu} \equiv \mathcal{Q}_{1}[\epsilon]+i \mathcal{Q}_{2}[\epsilon]
$$

are defined over a space-like surface $\Sigma$ whose area element is $d \Sigma_{\mu}$. Here $\mathcal{Q}_{I}$, $\mathrm{I}=1,2$, are the Majorana charge generators.

Now, imposing the gravitino field equation,

$$
\frac{2}{\kappa} \frac{\epsilon^{\mu \sigma \rho}}{e} \bar{\nabla}_{\sigma} \psi_{\rho}=\bar{\lambda} \gamma^{\mu}\left(\frac{1}{2} F_{\mu \nu} \sigma^{\mu \nu}+\frac{i \tilde{q}}{4}\left(|\phi|^{2}-v^{2}\right)\right)+\frac{1}{2} \bar{\chi} \gamma^{\mu}(\not D \phi)
$$

one can see from eqs.(48)-(49), after integration by parts, that the supercharge is nothing but the circulation of the gravitino arround the oriented boundary $\partial \Sigma$

$$
\mathcal{Q}[\epsilon]=-\frac{2}{\kappa} \oint_{\partial \Sigma} \bar{\epsilon} \psi_{\mu} d x^{\mu}
$$

Let us stress that the expression above coincides with the results presented by Teitelboim [13 for pure supergravity in 4-dimensional spacetime, after dimensional reduction. Although there are well-known problems for constructing supergravity charges in $2+1$ dimensions [28]-29], we shall see that they can be overcome in the present model.

As explained in [13], it is not possible to compute the supercharge algebra by (naively) evaluating Posson brackets from (51) because surface terms do 
not have well defined functional derivatives and hence their Poisson brackets with the various fields of the theory are not well defined. One can compute instead the algebra by acting on the integrand of (51) with a supersymmetry transformation:

$$
\{\overline{\mathcal{Q}}[\epsilon], \mathcal{Q}[\epsilon]\}\left|\equiv \delta_{\epsilon} \mathcal{Q}[\epsilon]\right|=\frac{2}{\kappa} \oint_{\partial \Sigma} \bar{\epsilon} \delta_{\epsilon} \psi_{\mu} d x^{\mu}=\frac{4}{\kappa^{2}} \oint_{\partial \Sigma} \bar{\epsilon} \hat{\nabla}_{\mu} \epsilon d x^{\mu}
$$

where $\overline{\mathcal{Q}}$ is given by:

$$
\overline{\mathcal{Q}}=\frac{2}{\kappa} \oint_{\partial \Sigma} \bar{\psi}_{\mu} \epsilon d x^{\mu}
$$

Now, Teitelboim [13 has proven, using Dirac formalism for constrained systems, that supergravity charges, which can be written as surface integrals in the form (51), obey a flat-space supersymmetry algebra

$$
\left\{\overline{\mathcal{Q}}_{I}, \mathcal{Q}_{J}\right\}=\delta_{I J} \gamma^{\mu} P_{\mu}+\epsilon_{I J} Z
$$

where $Z$ is the central charge. In flat space, it is a well-known result that this algebra leads in several models to Bogomol'nyi bounds for the energy [7]-11]. Indeed, squaring eq.(54) and tracing over the indices, one obtains a bound

$$
P^{2}-Z^{2} \geq 0
$$

from which, after using the identity of $Z$ with the topological charge of the field configuration T [7]-[11], one obtains the well-known Bogomol'nyi bound for the mass $M$ of the configuration

$$
M \geq|T|
$$

Coming back to supergravity, let us see explicitely how (52) ensures that static finite-energy configurations satisfy a bound of topological nature of the type (55). To this end, let us write, using the expression for the covariant derivative given in (32)

$$
\begin{aligned}
\{\overline{\mathcal{Q}}[\epsilon], \mathcal{Q}[\epsilon]\} \mid & =\frac{4}{\kappa^{2}} \oint_{\partial \Sigma} \bar{\epsilon} \hat{\nabla}_{\mu} \epsilon d x^{\mu} \\
& =\frac{4}{\kappa^{2}} \oint_{\partial \Sigma} \bar{\epsilon} \mathcal{D}_{\mu} \epsilon d x^{\mu}+i \oint_{\partial \Sigma} \bar{\epsilon} \epsilon\left(J_{\mu}+\tilde{q} v^{2} A_{\mu}\right) d x^{\mu}
\end{aligned}
$$


We can now use the asymptotic behaviour of different fields appearing in (56). The spinorial connection which enters in the covariant derivative in the first term of the r.h.s. behaves as

$$
\omega_{\bar{z}+-} \rightarrow \frac{\kappa^{2} M}{2 \bar{z}}
$$

Concerning the electromagnetic field as well as the Higgs current, finiteness of the energy implies

$$
A_{z} \rightarrow-\frac{i n}{\tilde{q} z} \quad, \quad A_{\bar{z}} \rightarrow \frac{i n}{\tilde{q} \bar{z}} \quad, \quad J_{z} \rightarrow O\left(\frac{1}{z \bar{z}}\right) \quad, \quad J_{\bar{z}} \rightarrow O\left(\frac{1}{z \bar{z}}\right) .
$$

(Here, the integer $n$ is the topological number that characterizes the quanta of magnetic flux). Finally, the asymptotic behaviour of $\epsilon$ will be written in the form:

$$
\epsilon \rightarrow \Theta(z \bar{z}) \epsilon_{\infty}
$$

where $\Theta(z \bar{z})$ will be determined using the so-called Witten condition 16 (see below).

We can now evaluate the line-integral (56). To avoid infrared divergences, it is necessary to take the contour of integration at large but finite radius $R$. Using the asymptotic behaviours listed above, (56) becomes:

$$
\{\overline{\mathcal{Q}}[\epsilon], \mathcal{Q}[\epsilon]\} \mid=\left(M \bar{\epsilon}_{\infty} \gamma^{0} \epsilon_{\infty}-v^{2} n \bar{\epsilon}_{\infty} \epsilon_{\infty}\right) \Theta(R)^{2}
$$

The relation of this result with the Poisson brackets (54) which are valid, in particular, in flat space, is, of course, no accident: as we discussed above, supersymmetry transformations at spatial infinity generate global $N=2$ supersymmetry algebra.

We can now prove that $\{\overline{\mathcal{Q}}[\epsilon], \mathcal{Q}[\epsilon]\} \mid$ is semi-positive definite and then derive a Bogomol'nyi bound of topological origin from (60).

First, let us observe that the supercharge algebra evaluated in the purely bosonic sector is the integral over the boundary of a 1-form $\omega$, constructed from a fermionic parameter:

$$
\begin{gathered}
\{\overline{\mathcal{Q}}[\epsilon], \mathcal{Q}[\epsilon]\} \mid=\frac{4}{\kappa^{2}} \oint_{\partial \Sigma} \omega \\
\omega=\bar{\epsilon} \hat{\nabla}_{\mu} \epsilon d x^{\mu}
\end{gathered}
$$


Now, $\omega$ can be identified with the generalized Nester-like form [16, 17] in 3 dimensional spacetime. The use of Nester form is at the root of several proofs of the positivity of gravitational energy [13]- [17] and it was also used in 4 and 5-dimensional models to find Bogomol'nyi bounds [19]- 22]. In the same vein, we shall see below that the integral of $\omega$ on the contour is semi-positive definite and that, as a consequence, the theory posses a Bogomol'nyi bound. Let us mention at this point that the integral in the r.h.s. of (61) coincides with the quantity $\Delta(r)$ introduced in Ref.[23]. Our construction shows that its occurence is a consequence of the $N=2$ supercharge algebra.

First, using Stokes' theorem, we have

$$
\oint_{\partial \Sigma} \omega=\int_{\Sigma} \epsilon^{\mu \nu \beta} \hat{\nabla}_{\beta}\left(\bar{\epsilon} \hat{\nabla}_{\mu} \epsilon\right) d \Sigma_{\nu} .
$$

where the integrand in (63) can be written as

$$
\epsilon^{\mu \nu \beta} \hat{\nabla}_{\beta}\left(\bar{\epsilon} \hat{\nabla}_{\mu} \epsilon\right)=\epsilon^{\mu \nu \beta} \hat{\nabla}_{\beta} \epsilon \hat{\nabla}_{\mu} \epsilon+\frac{1}{2} \epsilon^{\mu \nu \beta} \bar{\epsilon}\left[\hat{\nabla}_{\beta}, \hat{\nabla}_{\mu}\right] \epsilon .
$$

Then, using

$$
\left[\hat{\nabla}_{\mu}, \hat{\nabla}_{\nu}\right]=\frac{1}{2} R_{\mu \nu}{ }^{a b} \Sigma_{a b}+\frac{i \tilde{q} v^{2} \kappa^{2}}{2} F_{\mu \nu}+\frac{i \kappa^{2}}{2}\left(\partial_{\mu} J_{\nu}-\partial_{\nu} J_{\mu}\right),
$$

Einstein equations (37) and supersymmetric transformations for the fermionic fields $\lambda$ and $\chi$, we arrive to the following expression

$$
\epsilon^{\mu \nu \beta} \hat{\nabla}_{\beta}\left(\bar{\epsilon} \hat{\nabla}_{\mu} \epsilon\right)=\epsilon^{\mu \nu \rho} \overline{\hat{\nabla}_{\mu} \epsilon} \hat{\nabla}_{\rho} \epsilon+\frac{\kappa^{2}}{2}\left[\overline{\delta_{\epsilon} \lambda} \gamma^{\nu} \delta_{\epsilon} \lambda+\overline{\delta_{\epsilon} \chi} \gamma^{\nu} \delta_{\epsilon} \chi\right] .
$$

We now specialize our spacelike integration surface $\Sigma$ so that $d \Sigma_{\mu}=\left(d \Sigma_{t}, \overrightarrow{0}\right)$. Then, we only need to compute the time component of eq.(66) which, after some Dirac algebra, reads

$$
\begin{aligned}
\epsilon^{t \nu \beta} \hat{\nabla}_{\beta}\left(\bar{\epsilon} \hat{\nabla}_{\mu} \epsilon\right) & =\left(\gamma^{i} \hat{\nabla}_{i} \epsilon\right)^{\dagger}\left(\gamma^{j} \hat{\nabla}_{j} \epsilon\right)-g^{i j}\left(\hat{\nabla}_{i} \epsilon\right)^{\dagger}\left(\hat{\nabla}_{j} \epsilon\right) \\
& +\frac{\kappa^{2}}{2}\left[\delta_{\epsilon} \lambda^{\dagger} \delta_{\epsilon} \lambda+\delta_{\epsilon} \chi^{\dagger} \delta_{\epsilon} \chi\right]
\end{aligned}
$$

We see at this point, that if we impose the generalized Witten condition [16]

$$
\gamma^{i} \hat{\nabla}_{i} \epsilon=0
$$


the r.h.s. of eq.(67) is semi-positive definite

$$
\epsilon^{t \nu \beta} \hat{\nabla}_{\beta}\left(\bar{\epsilon} \hat{\nabla}_{\mu} \epsilon\right) \geq 0
$$

and then, using (63),

$$
\{\overline{\mathcal{Q}}[\epsilon], \mathcal{Q}[\epsilon]\} \mid \geq 0
$$

The bound is saturated if and only if

$$
\begin{aligned}
& \delta_{\epsilon} \lambda=0 \\
& \delta_{\epsilon} \chi=0 \\
& \hat{\nabla}_{i} \epsilon=0
\end{aligned}
$$

Condition (73) reflects our choice of surface $\Sigma$. One can easily see that a more general choice would imply, instead of (73),

$$
\hat{\nabla}_{\mu} \epsilon=0 \text {. }
$$

In explicit form, eqs.(71), (72) and (74) read:

$$
\begin{gathered}
\delta_{\epsilon} \lambda=\frac{1}{2}\left[F_{\mu \nu} \sigma^{\mu \nu}+\frac{i \tilde{q}}{2}\left(|\phi|^{2}-v^{2}\right)\right] \epsilon=0 \\
\delta_{\epsilon} \chi=\frac{1}{2}(\not D \phi) \epsilon=0 \\
\delta_{\epsilon} \psi_{\mu}=\left(\mathcal{D}_{\mu}+i \frac{\kappa^{2}}{4}\left(J_{\mu}+\tilde{q} v^{2} A_{\mu}\right)\right) \epsilon=0
\end{gathered}
$$

We can see at this point that solutions of (75) and (76) break half of the supersymmetry. Indeed, writting

$$
\epsilon \equiv\left(\begin{array}{c}
\epsilon_{+} \\
\epsilon_{-}
\end{array}\right)
$$

One can easily see that the conditions

$$
\begin{gathered}
\delta_{\epsilon_{+}} \lambda=0 \\
\delta_{\epsilon_{+}} \chi=0
\end{gathered}
$$


imply $\delta_{\epsilon_{-}} \lambda \neq 0, \delta_{\epsilon_{-}} \chi \neq 0$ for nontrivial solutions. Hence if one is to search Bogomol'nyi equations for non-trivial configurations, it makes sense to consider that $\epsilon$ has just one independent complex component,

$$
\epsilon \equiv\left(\begin{array}{c}
\epsilon_{+} \\
0
\end{array}\right)
$$

satisfying equation (68), which then reads

$$
\hat{\nabla}_{z} \epsilon_{+}=0
$$

Let us stress on the fact that field configuration solving Bogomol'nyi equations (which as we shall see coincide with (79) and (80)) break half of the supersymmetries, a common feature in all models presenting Bogomol'nyi bounds with supersymmetric extension (See for example [12, 20]). It can be understood as follows: The number of Killing spinors (those that solve eq.(77)) admitted by a certain spacetime coincides with the number of remnant unbroken supersymmetries [12]. If we attempt to keep all the supersymmetries of our model, we will find that the resulting field configuration has zero energy (the trivial vacuum) as it was remarked above. Then, it is evident that we must restrict the space of solutions of (77) if we want to obtain non-trivial topological configurations (which, in this sense, require breaking of one of the supersymmetries).

We can now study the asymptotic behaviour of the Killing spinor $\epsilon_{+} \rightarrow$ $\Theta(R) \epsilon_{+\infty}$. One can see that eq. (82) implies that $\Theta(R)$ is a power of the radius $R$ :

$$
\Theta(R)=R^{-\kappa^{2} \frac{n v^{2}}{2}}
$$

It is important, at this point, to comment on the existence of non-trivial solutions to eq. 82 in asymptotically conical spaces. In fact, the supercovariant derivative in (82) gets an electromagnetic contribution related to the magnetic flux, and then, as explained in [23], leads to the existence of non-trivial solutions, otherwise absent [16].

Now, for a parameter $\epsilon$ of the form (81), formula (60) can be written as

$$
\{\overline{\mathcal{Q}}[\epsilon], \mathcal{Q}[\epsilon]\} \mid=\left(M-v^{2} n\right) \epsilon_{+\infty}^{\dagger} \epsilon_{+\infty} \Theta(R)^{2}
$$

We can now finally write a Bogomol'nyi bound using (70) and (84):

$$
M-n v^{2} \geq 0
$$


The mass of our field configuration, defined in eq.(42), is then bounded from below by the magnetic flux quanta. Analogous results also hold in the 4 and 5 dimensional models studied by Kallosh et al [20] and Gibbons et al [21] where the bound reads: $M \geq \sqrt{Q^{2}+P^{2}}, Q$ and $P$ being the electric and magnetic charges respectively, related to the two central charges existing in the extended supersymmetry algebra of those models $[12$. In the present abelian 3-dimensional model there is just one central charge and, moreover, it is not possible to have electrically charged finite energy solitonic configurations in the Abelian Higgs model so that $Q=0$.

The bound (85) for the $d=3$ Abelian Higgs model coupled to gravity coincides with that presented in [23]. The novelty here is that we have obtained it from the supercharge algebra (plus the generalized Witten condition) thus showing that the connection between global supersymmetry and Bogomol'nyi bounds discussed in [7]-[11], also works for local supersymmetry.

The bound (85) is saturated whenever eqs.(75)-(77) hold and hence, we identify them as the Bogomol'nyi equations of our model. Concerning equations $(75)$ and (76), they can be written in the usual form [3]:

$$
\begin{gathered}
\epsilon^{z \bar{z}} F_{z \bar{z}}+\frac{\tilde{q}}{2}\left(|\phi|^{2}-v^{2}\right)=0 \\
D_{z} \phi=0 .
\end{gathered}
$$

They have been found previously in the study of the Einstein-Abelian Higgs model by Comtet and Gibbons in ref. 30 where it is shown that this set of equations admits vortex solutions in $3+1$ dimensions which can be interpreted as cosmic strings. Concerning eq.(77),

$$
\left(\mathcal{D}_{\mu}+i \frac{\kappa^{2}}{4}\left(J_{\mu}+\tilde{q} v^{2} A_{\mu}\right)\right) \epsilon_{+}=0
$$

it can be thought as the Bogomol'nyi equation for the gravitational field. Indeed, it can be seen that the integrability conditions of this equation,

$$
\left[\mathcal{D}_{\mu}+i \frac{\kappa^{2}}{4}\left(J_{\mu}+\tilde{q} v^{2} A_{\mu}\right), \mathcal{D}_{\nu}+i \frac{\kappa^{2}}{4}\left(J_{\nu}+\tilde{q} v^{2} A_{\nu}\right)\right] \epsilon_{+}=0
$$

become the Einstein equations once eqs.(86)-(87) are imposed. Even though conical spaces usually does not admit covariantly constant spinors, solutions 
to eq. (88) exist as a consequence of the electromagnetic contribution to the supercovariant derivative, as explained above. In view of the bound (85), the solutions of the whole set of Bogomol'nyi equations satisfy the more involved second order Euler-Lagrange ones.

\section{Summary and discussion}

We have studied Bogomol'nyi bounds for the Abelian Higgs model coupled to gravity in $2+1$ dimensions by embedding it in an extended $N=2$ supergravity model.

Bogomol'nyi equations for the scalar and the gauge field, compatible with the Einstein equation, were originally derived by Comtet and Gibbons [30], following the usual Bogomol'nyi approach [3]. In their work, the existence of gravitating multi-vortex solutions saturating the Bogomol'nyi bound was proven. These solutions can be understood as cosmic strings and the bound can be seen to be a restriction in possible values for the deficit angle of conical spacetime [30].

More recently, Becker, Becker and Strominger [23] considered the model we discussed and obtained the Bogomol'nyi bound from supersymmetry arguments. They followed an approach which is a variant of the methods leading to energy positivity in gravity models [16]-18. This approach is close to the one we have followed in the present paper. The novelty in our work is that we have obtained the Bogomol'nyi bound and the self-duality equations (including the one associated with the Einstein equation) by constructing the supercharge algebra and making explicit its relation with the (generalized) Witten-Nester-Israel form.

As it is well-known, in $2+1$ dimensions, states with non-zero energy produce asymptotically conical spaces and this put stringent limitations on the existence of covariantly constant spinors which are basic in the construction of supercharges. However, in our model, the supercovariant derivative gets a contribution related to the topological charge of the vortex and this allows for non-trivial solutions for Killing spinors. This is the reason why we were able to find supercharges and determine their algebra.

A possible interest of our investigation is related to the recent discussion about how can supersymmetry ensure the vanishing of the cosmological constant [28], 23], 29]. On the other side, since our approach provides a sys- 
tematic way of investigating Bogomol'nyi bounds in supergravity models in arbitrary dimensions (as it is the case for global supersymmetry [7]-[11]), one can think in finding Bogomol'nyi bounds for other gauge models coupled to gravity like, for example, the Chern-Simons-Higgs model recently considered in [31]. We hope to come back to these problems in a forthcoming work.

\section{References}

[1] R. Rajaraman, Solitons and Instantons, North Holland (1982).

[2] A.A. Belavin, A.M. Polyakov, A.S. Schwartz and Yu.S. Tyupkin, Phys. Lett. B 59 (1975) 85.

[3] E.B. Bogomol'nyi, Sov. Jour. Nucl. Phys. 24 (1976) 449.

[4] H. de Vega and F.A. Schaposnik, Phys. Rev. D 14 (1976) 1100.

[5] A. Salam and J. Strathdee, Nucl. Phys. B 97 (1975) 293;

P.Fayet, Il Nuovo Cimento A 31 (1976) 626.

[6] P. Di Vecchia and S. Ferrara, Nucl. Phys. B 130 (1977) 93.

[7] E. Witten and D. Olive, Phys. Lett. B 78 (1978) 77.

[8] Z. Hlousek and D. Spector, Nucl. Phys. B 370 (1992) 43.

[9] Z. Hlousek and D. Spector, Nucl. Phys. B 397 (1993) 173; Phys. Lett. B 283 (1992) 75; Mod. Phys. Lett. A 7 (1992) 3403.

[10] C. Lee, K. Lee and E.J. Weinberg, Phys.Lett.B243(1990)105.

[11] J.D. Edelstein, C. Núñez and F.A. Schaposnik, Phys. Lett. B 329 (1994) 39.

[12] C.M. Hull, Nucl.Phys.B239(1984)541.

[13] C. Teitelboim, Phys. Lett. B 69 (1977) 240

[14] S. Deser and C. Teitelboim, Phys. Rev. Lett. 39 (1977) 249. 
[15] M. Grisaru, Phys. Lett. B 73 (1978) 249.

[16] E. Witten, Comm. Math. Phys. 80 (1981) 381.

[17] J.M. Nester, Phys. Lett. A 83 (1981)241;

W. Israel and J.M. Nester, Phys. Lett. A 85 (1981)259

[18] G.W. Gibbons and C.M. Hull, Phys. Lett. B 109 (1982)190.

[19] G.W. Gibbons, C.M. Hull and N.P.Warner, Nucl.Phys.B218(1983)173;

D.Z.Freedman and G.W. Gibbons, Nucl.Phys.B233(1984)24.

[20] R. Kalosh, A. Linde, T. Ortin and A. Peet, Phys. Rev. D 46 (1992) 5278.

[21] G.W. Gibbons, D. Kastor, L.A.J. London, P.K. Townsend and J. Traschen, Nucl.Phys.B416(1994)850.

[22] M. Cvetič, S. Griffies and S.-J. Rey, Nucl.Phys.B381(1992)301.

[23] K. Becker, M. Becker and A. Strominger, "Three-Dimensional Supergravity And The Cosmological Constant", hep-th/9502107.

[24] E. Cremmer and B. Julia, Nuc.Phys.B159(1979)141;

J. Scherk and J.H. Schwartz, Phys.Lett.82B(1979)60.

[25] J. Wess and J. Bagger, "Supersymmetry and Supergravity", Princeton University Press (1990).

[26] S. Ferrara, L. Girardello, T. Kugo and A. van Proeyen, Nucl.Phys.B223 (1983)191.

[27] See for example R. Jackiw, Five Lectures on Planar Gravity, in Cocoyoc 1990, Proceedings, Relativity and Gravitation: Classical and quantum, p.74 Mexico (1991).

[28] E. Witten, Int. J. Modern Physics A10 (1995) 1247.

[29] E. Witten, "Strong Coupling and the Cosmological Constant", hep-th /9506101. 
[30] A. Comtet and G.W. Gibbons, Nucl.Phys.B299(1988)719.

[31] L.A.J. London, "Chern-Simons Vortices Coupled to Gravity" (to appear in Phys.Lett.B);

M.E.X. Guimaraẽs and L.A.J. London, "Non-Abelian, Self-Dual ChernSimons Vortices coupled to gravity", hep-th /9506050 